\title{
Chemical composition of ultrasonic-assisted $n$-hexane extracts of Sideritis scardica Grieseb. and Sideritis raeseri Boiss. \& Heldr. (Lamiaceae) from Macedonia and Albania
}

\author{
Bujar Qazimi, Marija Karapandzova*, Gjose Stefkov, Svetlana Kulevanova \\ Institute of Pharmacognosy, Faculty of Pharmacy, University SS Cyril and Methodius, Skopje, Republic of Macedonia \\ Received: May 2011; Accepted: June 2011
}

\begin{abstract}
Chemical composition of $n$-hexane extracts obtained from dried over-ground parts of two species of Sideritis, S. scardica Grieseb. and S. raeseri Boiss. \& Heldr. (Lamiaceae) was analyzed using GC/FID/MS. The collection of plants was made on different locations in the western part of Macedonia and the southern part of Albania, comprising twelve different samples of plant material. The ultrasonic-assisted extraction process was used for preparation of the $n$-hexane extracts yielded $0.73-3.33 \%$ and $9.11-10.44 \%$ of extracts for $S$. scardica and $S$. raeseri, respectively. Over one hundred constituents of the extracts were identified, belonging to several classes of components: diterpenes, hydrocarbons, dominantly present in each of the extracts, followed by fatty acids, aliphatic and aromatic alcohols, sterols, triterpene alcohols, and monoterpenes and sesquiterpenes, which were found in much smaller amounts or only in traces. The most abundant constituents of the extracts of both species of Sideritis were two diterpene components, both with $\mathrm{M}=286$, which were not fully identified. Large percentages of nonacosane (1.71-12.22\% and 7.46-19.68\% for S. scardica and S. raeseri, respectively) and hentriacontane (4.48-20.79\% and $8.09-30.31 \%$ for $S$. scardica and $S$. raeseri, respectively) were also found in the extracts of both species.
\end{abstract}

Key words: $\quad$ Sideritis scardica, Sideritis raeseri, $n$-hexane extracts, ultrasonic-assisted extraction, composition, GC-MS analysis.

\section{Introduction}

Sideritis scardica Griseb. and Sideritis raeseri Boiss. \& Heldr. (Lamiaceae) are wild, hardy flowering perennials that have adapted to survive with little water and little soil, also known as Mountain tea, Ironwort, Shepard's tea or Pirin tea. Both plants are very popular in Macedonia, Bulgaria, and Greece as well as throughout the Eastern Mediterranean as refreshment but also to cure common cold. The herbs are often used to prepare teas widely believed to alleviate sinus congestion, aches, pains and viruses including flu and common cold. A very aromatic variant of the tea includes combining the Sideritis plant with spices common to the Mediterranean cuisine. Scientists recently suggested that the traditional Greek promotion of mountain tea as a panacea for most illnesses may be remarkably close to the truth.

*Marija_Karapandzova@ff.ukim.edu.mk; marijakarapandzova@yahoo.com
In past decades species of genus Sideritis, especially species from Spain, Turkey, Bulgaria, Albania, Greece, and Serbia, have been extensively investigated on chemical composition of flavonoids, phenilpropanoids, cinnamic acid derivates and phenilethanoids (Palomino et al., 1996; Aboutabl et al., 2002; Ozkan et al., 2005; Erkan et al., 2011; Alipieva et al., 2010; Koleva et al., 2003; Gabrieli et al., 2005, Armata et al., 2008; Pljevljakusic et al., 2011) as well as on essential oils composition, chemistry of diterpeneoids, iridoids and other terpenoids or related components (Gomez-Serranillos et al., 1997; Gomez-Serranillos et al., 2004; Rodriguez-Garcia et al., 2004; Topku et al., 2001; Topku et al., 2002; Alipieva et al., 2009). Recently, data on flavonoids, phenilethanoids and phenilpropanoid acids and their esters have been reported for native Macedonian Sideritis (S. scardica and S. raeseri) (Janeska et al., 2007; Petreska et al., 2011a, 2011b), as well as data on essential oil composition of the same species (Kostadinova et al., 2007). 
In most of the studies conducted on Sideritis, scientists suggested that phenolic and/or polyphenolic components are responsible mostly for the antioxidant activity of alcohol extracts, while terpene components from the essential oils and even more different diterpeneoids are found to be responsible for the anti-inflammatory, analgesic, antiulcer, antibacterial, antifungal, cytotoxic and antitumor activity (Gomez-Serranillos et. al., 2004). Therefore, preparation of an $n$-hexane extracts of dried herbs of Sideritis is the most preferable type of extraction if terpenoid components represent research interest (Alcaraz et al., 1989; Gomez-Serranillos et al., 1998; Gomez-Serranillos et al., 1997; Aboutabl et al., 2002; Menghini et al., 2005; Tsaknis and Lalas, 2005; Kostadinova E. et al., 2008). Usually, the process of extraction is carried out via methods of percolation and maceration, characterized by small efficiency and long drawing (Lysyansky and Grebenyuk, 1987). Nowadays, the use of different electro-physical methods (ultrasound, electro-flotation, etc) allows increasing the extraction rate and yield of biologically active compounds (Sulman et al., 1997; Semagina et al., 2000).

Taking into account the consideration mentioned above, the aim of this work was preparation of $n$-hexane extract of Sideritis scardica and $S$. raeseri by ultrasonic-assistance extraction and determination of the chemical composition of the extracts using GC/FID/MS method.

\section{Experimental}

\subsection{Plant material}

Samples of Sideritis were collected in a flowering stage in the western, central and southern areas in R. Macedonia and southeastern parts of Albania. Sideritis scardica was collected from 7 different regions in Macedonia: Shar Planina (Ljuboten) (V.No. S1/08), Suva Gora (near Gostivar) (V.No. S2/08), Prilep (cultivated plant, V. No. S3/08), Bistra (Lazaropole) (V.No. S4/08), Kozuf (Dve ushi) (V.No. S5/08), Nidze (Kajmakchalan) (V. No. 14/08) and Ilina mountain (S 16/08), while the samples of Sideritis raeseri were collected from Galicica mountain (V. No. 15/08) and Baba mountain (V.No. S6/08) in Macedonia and from Gramoz mountain (V. No. S7/08), Tepelena (V. No. S8/08) and Logora mountain (V. No. S18.08) from south Albania. The collections were made in 2008 and 2009. A voucher specimen of each species was deposited in the Herbarium of the Pharmacognosy Department, Faculty of Pharmacy, University SS Cyril and Methodius, Skopje, Republic of Macedonia. Plant material was left to dry at room temperature and, after draying it was packed in paper bags and left in dark, cool and dry place until analysis.

\subsection{Extraction}

Plant extracts were obtained using ultrasonic-assisted extraction process at room temperature. $n$-Hexane was used for the extraction in 2 portions in ratio to plant material 1:20 (1 g plant material was extracted twice with $10 \mathrm{ml}$ solvent). The duration of the extraction was $2 \times 30$ minutes. The extracts obtained after filtration were concentrated to dryness below $35^{\circ} \mathrm{C}$. The residue were measured as yield of the extraction and then dissolved in $n$-hexane to obtained solution with concentration of $1 \mathrm{~g}$ plant material in $1 \mathrm{ml}$ solution. For GC/FID/MS analysis additional $1 \mu \mathrm{g} / \mathrm{ml}$ dilutions were prepared.

\subsection{Gas chromatography}

$n$-Hexane extracts were analyzed on Agilent 7890A Gas Chromatography system equipped with FID detector and HP-5ms 5\% phenyl 95\% dimethylpolysiloxane bonded phase capillary column (30 m x $0.25 \mathrm{~mm}$, film thickness $0.25 \mu \mathrm{m})$. Operating conditions were as follows: oven temperature at $60^{\circ} \mathrm{C}$ for $5 \mathrm{~min}$, then increased to $80^{\circ} \mathrm{C}$ at rate of $1^{\circ} \mathrm{C} / \mathrm{min}$ and held $2 \mathrm{~min}$ and at the end increased to $280^{\circ} \mathrm{C}$ at rate of $5^{\circ} \mathrm{C} / \mathrm{min}$ and held $5 \mathrm{~min}$; helium as carrier gas at a flow rate of $1 \mathrm{ml} / \mathrm{min}$; temperature of the injector $260^{\circ} \mathrm{C}$ and that of the FID detector $270^{\circ} \mathrm{C}$; the GC split ratio $1: 1$. $1 \mu \mathrm{l}$ of each sample of the essential oil, dissolved in xylene (1: $1000 \mathrm{v} / \mathrm{v}$ ) was injected per GC run.

The percentage composition of $n$-hexane extracts were computed by the normalization method from the GC/FID peak areas (relative amount of each compound to the total amount present), calculated by means of three injections from each extract.

The extracts were analyzed on Agilent 7890A Gas Chromatography system interfaced to an Agilent 5975C mass spectrometer. The gas chromatographic conditions were the same as reported for GC analysis and the same column was used. The mass spectrometry conditions were: ionization voltage $70 \mathrm{eV}$, ion source temperature $230^{\circ} \mathrm{C}$ and mass range from 50 - $500 \mathrm{Da}$

Identification of the components present in essential oils was made by comparing mass spectra of components in essential oils with those from Nist, Wiley and Adams mass spectra libraries, by AMDIS (Automated Mass Spectral Deconvolution and Identification System) and by comparing literature and estimated Kovat's (retention) indices that were determined using mixture of homologous series of normal alkanes from $\mathrm{C}_{9}$ to $\mathrm{C}_{25}$ in hexane, under the same above mentioned conditions.

\section{Results and discussion}

The ultrasonic-assisted extraction yielded different amount of dry extracts (dry residues after evaporation) ranged from $0.73-3.33 \%$ for the samples of $S$. scardica and from $9.11-10.44 \%$ for the samples of $S$. raeseri (Table 1.). The yields of $n$-hexane extracts of $S$. raeser $i$ were almost the same for different samples of the plant regardless of the collection site. In the case of $S$. scardica much more variations in the extraction yields appeared. The higher yield of 
Table 1. The yields of $n$-hexane extracts of Sideritis obtained by ultrasonic-assisted extraction

\begin{tabular}{lclc}
\hline \hline \multicolumn{1}{c}{ S. scardica } & $n$-hexane extr. (\%) & \multicolumn{1}{c}{ S. raeseri } & $n$-heksane extr. (\%) \\
\hline Shar Planina (Ljuboten) & 0.73 & Baba mountain. & 9.11 \\
Suva Gora (Gostivar) & 0.89 & Galicica mountain & 9.87 \\
Prilep (cultivated) & 1.78 & Gramoz mountain (Albania) & 10.44 \\
Bistra (Lazaropole) & 3.33 & Tepelena (Albania) & 9.53 \\
Kozuf (Dve ushi) & 0.82 & Logora mountain (Albania) & 9.34 \\
Nidze (Kajmakcalan) & 1.23 & & \\
Ilina mountain & 1.52 & & \\
\hline
\end{tabular}

$(\mathrm{n}=3)$

$n$-hexane extract for $S$. raeseri against $S$. scardica, probably due to the larger amounts of components extractible in hexane in $S$. raeseri. Bulgarian scientists reported 0.50 $0.58 \%$ yields of the $n$-hexane extracts for both species, $S$. scardica and $S$. raeseri, which is significantly lower from our findings (Kostadinova et al., 2008). The reason of lower yield probably lays in the differences in geographical origin of the samples, but it is worth mentioning that those scientists have used $24 \mathrm{~h}$ maceration as extraction procedure while in our case it was ultrasonic-assisted extraction. According to literature data, ultrasonic-assisted extraction has been pointed out as more effective extraction procedure (Sulman et al., 1997, Samagina et al., 2000).

Gas-chromatography analysis showed presence of over 100 individual components identified by GC/MS. The results of chemical composition of $n$-hexane extract of $S$. scardica are presented in Table 2 and for $S$. raeseri in Table 3 . The chemical composition of the $n$-hexane extracts of both species showed qualitative and quantitative similarities. Mono and sesquiterpenes were presented in very small amounts, almost in traces, but apart from that, 25 components were identified. In S. scardica the sesquiterpene: trans-caryophyllene, $\delta$-cadinene, caryophyllene oxide and $\alpha$-cadinol $+\tau$ muurolol denoted the main part of this fraction. In $S$. raeseri additionaly $\alpha$ - and $\beta$-pinenes, myrtenol, trans-cadina-1,4diene, viridiflorol, valeranone and $\alpha$-bisabolole were found. These data differ a lot from the previously reported data of chemical composition of essential oils of S. scardica (Galicnik) and $S$. raeseri (Galicica) from Macedonia (Kostadinova et. al., 2007). For both species, $\alpha$-cadinol was defined as the dominant component and elemol acetate and germacron additionaly for the $S$. raeseri. Interesting results for the chemical composition of essential oils of cultivated $S$. raeseri subsp. raeseri were recently reported for the plant cultivated in Serbia. The main mono and sesquiterpene components of those oils were bicyclogermacrene and spatulenol (Plevljakisic et al., 2011). In both cases essential oils were obtained by hydro-distillation and besides mono and sesquiterpenes contained fatty acids and their esters, hydrocarbons and several diterpenes. All these components were also found in $n$ hexane extracts of our specimens of Sideritis.
The fractions of fatty acids and fatty acid-ester compounds were found in larger amount than fractions of mono and sesqiterpenes. Within these fractions, the main components were methyl, isopropyl hexadecanoate and hexadecanoic acid in S. scardica as well as octadecanoic acid methyl ester and hexadecanoic acid in $S$. raeseri. Large fraction of the $n$-hexane extracts belonged to hydrocarbons comprising aliphatic components with long carbon-chains (C5-C33). The hydrocarbons represented 13.33-47.24\% of $n$-hexane extract of S. scardica (Fig. 1) and 26.24-68.12\% of $n$-hexane extracts of $S$. raeseri (Fig. 2). The most abundant components were heptacosane, nonacosane, hentriacontane and tritriacontane. Among them, hentriacontane was the predominant hydrocarbon in the extracts of both species, with 4.48-20.79 \% in S. scardica and 8.09-30.31 in $S$. raeseri. Very small part of extracts contained oxidized products of hydrocarbons such as different alcohols, rarely some esters. Kostadinova et al. (2008) in $n$-hexane extracts of $S$. scardica and $S$. scardica $x$ S. syriaca hybride also found nonacosane and hentriacontane as predominant hydrocarbons. Seasonal variation in the content of some hydrocarbons, mainly hentriacontane and nonacosane, was found for both species (Table 2 and 3 ).

Besides hydrocarbons, second predominant fraction, of the $n$-hexane extracts of the investigated species of Sideritis, were diterpeneoids. This fraction represented 23.00-60.80\% of the S. scardica (Fig. 1) and 22.13$45.44 \%$ of the $S$. raeseri $n$-hexane extracts (Fig. 2). $(+)$-Beyeren, pimara-8(14),15-diene, kaur-15-en, 7-ethenyl-1,2,3,4,4a,4b,5,6,7,9,10,10a-dodecahydro-1,1,4a,7tetramethyl-2-phenanthrenol and manyol oxide were identified an in addition eight more components were tentatively indetified as diterpeneoids. We were unable to identify the two dominant components in this fraction (in some cases the most dominant components in the whole extract). These components, both with $\mathrm{M}=286$, are probably pimarane derivates. Besides them, there were three diterpenee components with $\mathrm{M}=306, \mathrm{M}=346$ and $\mathrm{M}=286$, also not identified. Few of them were probably components with kaurane skeleton, as kauranes were often identified until now in different species of Sideritis as the predominant 
Table 2. Chemical composition of hexane extracts of Sideritis scardica from different location from Macedonia

\begin{tabular}{|c|c|c|c|c|c|c|c|c|c|c|c|c|}
\hline \multirow[b]{2}{*}{$\begin{array}{l}\text { MONO and } \\
\text { SESQUITERPENS }\end{array}$} & \multirow[t]{2}{*}{ KIE } & \multicolumn{2}{|c|}{$\begin{array}{l}\text { Shar Planina } \\
20082009\end{array}$} & \multicolumn{2}{|c|}{$\begin{array}{l}\text { Suva Gora } \\
20082009\end{array}$} & \multicolumn{2}{|c|}{$\begin{array}{l}\text { Prilepsko** } \\
20082009\end{array}$} & \multicolumn{2}{|c|}{$\begin{array}{c}\text { Bistra } \\
20082009\end{array}$} & \multirow[t]{2}{*}{$\begin{array}{c}\text { Kozuf } \\
2008\end{array}$} & \multirow[t]{2}{*}{$\begin{array}{l}\text { Nidze } \\
2008\end{array}$} & \multirow[t]{2}{*}{$\begin{array}{l}\text { Ilina Mtn. } \\
2008\end{array}$} \\
\hline & & & & & & & & & & & & \\
\hline$\alpha$-Pinene & 951.1 & $\operatorname{tr}$ & $\operatorname{tr}$ & - & - & - & - & 0.01 & - & - & $\operatorname{tr}$ & - \\
\hline Camphene & 960.7 & - & - & - & - & - & - & 0.01 & - & - & - & - \\
\hline$\beta$-Pinene & 980.8 & $\operatorname{tr}$ & - & - & - & - & - & 0.01 & - & - & - & - \\
\hline$\beta$-Myrcene & 992.6 & - & - & - & - & - & - & 0.01 & - & - & - & - \\
\hline $\begin{array}{l}\text { D-Limonene }+ \\
\beta \text {-Phellandren }\end{array}$ & 1024.2 & $\operatorname{tr}$ & $\operatorname{tr}$ & $\operatorname{tr}$ & $\operatorname{tr}$ & - & - & 0.02 & $\operatorname{tr}$ & $\operatorname{tr}$ & $\operatorname{tr}$ & $\operatorname{tr}$ \\
\hline trans-Pinocarveole & 1145.1 & $\operatorname{tr}$ & - & $\operatorname{tr}$ & $\operatorname{tr}$ & $\operatorname{tr}$ & $\operatorname{tr}$ & - & - & - & - & - \\
\hline trans-Sabinol & 1152.9 & $\operatorname{tr}$ & - & $\operatorname{tr}$ & $\operatorname{tr}$ & $\operatorname{tr}$ & - & - & - & - & $\operatorname{tr}$ & \\
\hline Myrtenol & 1212.9 & 0.05 & 0.02 & $\operatorname{tr}$ & $\operatorname{tr}$ & $\operatorname{tr}$ & $\operatorname{tr}$ & 0.01 & $\operatorname{tr}$ & $\operatorname{tr}$ & $\operatorname{tr}$ & $\operatorname{tr}$ \\
\hline (-)-Verbenone & 1231.5 & $\operatorname{tr}$ & - & $\operatorname{tr}$ & $\operatorname{tr}$ & 0.06 & $\operatorname{tr}$ & 0.01 & $\operatorname{tr}$ & - & $\operatorname{tr}$ & - \\
\hline$\alpha$-Cubebene & 1403.6 & 0.02 & & - & $\operatorname{tr}$ & $\operatorname{tr}$ & - & - & - & - & $\operatorname{tr}$ & - \\
\hline$\alpha$-Copaene & 1437.4 & - & 0.01 & $\operatorname{tr}$ & $\operatorname{tr}$ & $\operatorname{tr}$ & - & - & 0.05 & $\operatorname{tr}$ & 0.07 & 0.05 \\
\hline Muurola-4(14),15-diene (cis) & 1454.2 & 0.03 & - & - & - & - & - & - & - & - & - & - \\
\hline trans-Caryophyllene & 1488.7 & 0.09 & 0.10 & 0.14 & 0.26 & 0.05 & 0.08 & 0.01 & 0.07 & 0.30 & 0.31 & 0.08 \\
\hline Muurola-3,5-diene (trans) & 1524.7 & - & - & $\operatorname{tr}$ & $\operatorname{tr}$ & $\operatorname{tr}$ & - & - & - & - & - & - \\
\hline$\alpha$-Hummulene & 1528.9 & 0.01 & - & - & - & - & - & - & $\operatorname{tr}$ & 0.07 & 0.05 & - \\
\hline Germacren D & 1560.7 & 0.03 & 0.01 & - & - & - & - & 0.01 & $\operatorname{tr}$ & 0.04 & 0.05 & 0.08 \\
\hline Bicyclogermacrene & 1578.1 & 0.03 & 0.01 & - & - & - & - & - & - & - & - & - \\
\hline$\delta$-Cadinene & 1606.4 & 0.05 & 0.02 & - & - & - & - & $\operatorname{tr}$ & 0.08 & - & 0.15 & 0.03 \\
\hline Cadina-1,4-diene (trans) & 1617.3 & 0.04 & 0.02 & - & - & 0.06 & - & $\operatorname{tr}$ & - & - & 0.04 & 0.05 \\
\hline (-)-Spatulenol & 1669.8 & 0.05 & 0.02 & & & - & - & $\operatorname{tr}$ & - & 0.04 & 0.04 & - \\
\hline Caryophyllene oxide & 1677.0 & 0.12 & 0.10 & 0.13 & 0.12 & - & 0.11 & - & 0.04 & 0.05 & 0.12 & 0.07 \\
\hline Viridiflorol & 1686.1 & 0.03 & 0.01 & - & - & - & - & - & 0.05 & - & - & - \\
\hline $\begin{array}{l}\alpha \text {-Cadinol }+ \\
\tau-\text { Muurolol }\end{array}$ & $\begin{array}{l}1739.1 \\
1739.5\end{array}$ & 0.07 & 0.05 & - & - & - & - & - & 0.30 & 0.04 & 0.03 & 0.05 \\
\hline Valeranone & 1775.4 & - & 0.03 & - & - & - & - & $\operatorname{tr}$ & $\operatorname{tr}$ & - & - & 0.03 \\
\hline $\begin{array}{l}\text { Total mono- and } \\
\text { sesquiterpens: }\end{array}$ & & 0.71 & 0.43 & 0.35 & 0.47 & 0.30 & 0.22 & 0.21 & 0.67 & 0.57 & 0.95 & 0.49 \\
\hline \multicolumn{13}{|l|}{ DITERPENS } \\
\hline$(+)$-Beyerene & 2056.7 & 0.07 & 0.05 & - & - & 0.06 & - & 0.01 & $\operatorname{tr}$ & - & - & - \\
\hline Pimara-8(14),15-diene & 2072.4 & 0.07 & 0.05 & - & - & - & - & $\operatorname{tr}$ & - & - & - & - \\
\hline Kaur-15-en & 2126.0 & 0.10 & 0.08 & - & & 0.16 & - & 0.05 & 0.07 & 0.05 & - & - \\
\hline Manoyl oxide & 2146.2 & 0.04 & 0.02 & - & & - & - & - & $\operatorname{tr}$ & - & - & - \\
\hline $\begin{array}{l}\text { 3 } \alpha \text {-Hydroxymanool } \\
(\mathrm{M}=288)^{*}\end{array}$ & 2327.3 & 0.22 & 0.20 & 0.24 & 0.09 & 0.23 & 0.21 & 0.08 & 0.29 & 0.08 & 0.06 & 0.06 \\
\hline $\begin{array}{l}\text { 7-ethenyl- } \\
\text { 1,2,3,4,4a,4b,5,6,7,9,10,10a- } \\
\text { dodecahydro-1,1,4a,7- } \\
\text { tetramethyl-2-phenanthrenol } \\
(\mathrm{M}=306)\end{array}$ & 2335.8 & 0.26 & 0.24 & 0.23 & 0.24 & 0.23 & 0.13 & 0.08 & 0.18 & 0.13 & 0.10 & 0.15 \\
\hline
\end{tabular}




\begin{tabular}{|c|c|c|c|c|c|c|c|c|c|c|c|c|}
\hline Components & KIE & \multicolumn{2}{|c|}{$\begin{array}{l}\text { Shar Planina } \\
20082009\end{array}$} & \multicolumn{2}{|c|}{$\begin{array}{l}\text { Suva Gora } \\
20082009\end{array}$} & \multicolumn{2}{|c|}{$\begin{array}{l}\text { Prilepsko** } \\
20082009\end{array}$} & \multicolumn{2}{|c|}{$\begin{array}{c}\text { Bistra } \\
20082009\end{array}$} & \multirow{2}{*}{$\begin{array}{c}\begin{array}{c}\text { Kozuf } \\
2008\end{array} \\
0.07\end{array}$} & \multirow{2}{*}{$\begin{array}{c}\text { Nidze } \\
2008 \\
-\end{array}$} & \multirow{2}{*}{$\begin{array}{c}\text { Ilina Mtn. } \\
2008 \\
0.10\end{array}$} \\
\hline Diterpene $(\mathrm{M}=306)$ & 2374.2 & 0.22 & 0.19 & 0.12 & 0.13 & 0.19 & - & 0.07 & 0.09 & & & \\
\hline Diterpene $(\mathrm{M}=306)$ & 2388.5 & - & - & - & - & 0.24 & - & 0.06 & 0.19 & 0.15 & 0.04 & 0.12 \\
\hline Diterpene $(\mathrm{M}=306)$ & 2458.8 & 0.64 & 0.62 & 0.28 & 0.22 & 0.76 & 0.28 & 0.25 & 0.44 & 0.46 & 0.23 & 0.14 \\
\hline Diterpene $(\mathrm{M}=286)^{*}$ & 2487.7 & 10.27 & 10.49 & 6.70 & 7.78 & 13.14 & 6.11 & 8.72 & 12.65 & 8.52 & 4.15 & 4.68 \\
\hline Diterpene $(\mathrm{M}=286)^{*}$ & & 36.02 & 37.12 & 24.08 & 28.25 & 42.43 & 19.92 & 15.86 & 31.15 & 28.66 & 20.00 & 17.04 \\
\hline Diterpene $(\mathrm{M}=346) *$ & - & - & 1.32 & - & 0.92 & - & 0.89 & 0.67 & 0.30 & 0.92 & 0.55 & 0.59 \\
\hline Diterpen $(\mathrm{M}=286) *$ & - & - & - & 0.74 & 1.86 & 3.59 & 1.50 & 1.13 & 2.23 & 2.02 & 1.47 & 0.11 \\
\hline Total diterpens: & & 48.91 & 50.38 & 32.31 & 39.49 & 61.03 & 29.04 & 38.04 & 47.61 & 41.06 & 27.50 & 23.15 \\
\hline ESTERS & & & & & & & & & & & & \\
\hline Benzyl benzoate & 1924.3 & 0.05 & 0.02 & - & - & - & 0.05 & $\operatorname{tr}$ & 0.04 & - & - & - \\
\hline Isopropyl tetradecanoate & 1978.5 & 0.05 & 0.03 & 0.08 & - & 0.04 & 0.11 & - & 0.08 & 0.06 & 0.25 & 0.32 \\
\hline Di-isobutyl phthalate* & 2029.2 & 0.05 & 0.01 & - & - & - & 0.09 & $\operatorname{tr}$ & 0.07 & - & - & - \\
\hline Methyl hexadecanoate & 2103.3 & 0.05 & 0.03 & - & - & - & 0.11 & 0.01 & 0.06 & 0.04 & - & - \\
\hline Hexadecanoic acid ethylester & 2135.6 & - & - & - & & 0.06 & - & 0.03 & 0.07 & - & - & - \\
\hline Isopropyl hexadecanoate & 2243.4 & - & 0.03 & - & - & - & - & - & 0.04 & - & - & - \\
\hline Methyl octadecanoate* & & - & 0.10 & 0.10 & 0.14 & - & 0.12 & 0.04 & 0.05 & - & - & - \\
\hline $\begin{array}{l}\text { 9,12-Octadienoic acid } \\
\text { ethylester* }\end{array}$ & & - & - & - & - & - & - & - & 0.10 & 0.15 & 0.10 & 0.15 \\
\hline Total esters: & & 0.20 & 0.22 & 0.18 & 0.14 & 0.10 & 0.48 & 0.14 & 0.52 & 0.25 & 0.35 & 0.47 \\
\hline $\begin{array}{l}\text { HYDROCARBONS } \\
\text { Heptadecane }\end{array}$ & 1790.5 & 0.05 & 0.01 & - & 0.10 & - & - & 0.22 & 0.03 & 0.05 & - & - \\
\hline Octadecane & 1896.4 & $\operatorname{tr}$ & 0.01 & - & - & - & - & - & 0.02 & - & - & - \\
\hline Nonadecane & 2002.0 & 0.03 & 0.01 & - & - & - & - & 0.03 & 0.04 & 0.04 & 0.05 & - \\
\hline Eicosane & 2108.2 & 0.06 & 0.04 & - & - & - & 0.13 & - & 0.03 & - & 0.03 & 0.03 \\
\hline Heneicosane & 2213.4 & 0.07 & 0.05 & - & - & - & 0.09 & $\operatorname{tr}$ & 0.06 & 0.04 & 0.06 & 0.06 \\
\hline Docosane & 2318.5 & 0.16 & 0.14 & - & - & 0.05 & 0.18 & - & 0.08 & - & 0.15 & 0.10 \\
\hline Tricosane & 2423.2 & 0.27 & 0.25 & 0.57 & 0.39 & 0.38 & 0.32 & 0.11 & 0.35 & 0.26 & 0.59 & 0.31 \\
\hline Tetracosane & 2528.7 & 0.07 & 0.05 & 0.14 & 0.10 & 0.16 & 0.36 & 0.04 & 0.41 & 0.10 & 0.18 & 0.09 \\
\hline Pentacosane & 2631.0 & 0.36 & 0.34 & 0.82 & 0.64 & 0.80 & 0.82 & 0.20 & 0.90 & 0.79 & 1.47 & 0.84 \\
\hline Hexacosane & 2691.5 & - & 0.07 & - & - & - & 0.09 & - & 0.12 & - & 0.04 & 0.07 \\
\hline Heptacosane & 2784.4 & 1.66 & 1.62 & 3.17 & 2.73 & 1.68 & 3.12 & 0.47 & 2.19 & 2.17 & 4.10 & 3.86 \\
\hline Octacosen* & 2810.3 & - & 0.27 & - & 0.44 & 0.56 & 0.68 & 0.08 & 0.48 & 0.18 & 0.55 & 0.18 \\
\hline Octacosane & 2822.2 & 0.30 & 0.28 & 0.46 & 0.49 & 0.50 & 0.72 & 0.11 & 0.72 & 0.52 & 0.61 & 0.52 \\
\hline Nonacosane & 3024.6 & 7.42 & 7.41 & 12.22 & 10.41 & 3.48 & 11.01 & 1.71 & 7.20 & 9.59 & 0.13 & 8.59 \\
\hline Triacontane & 3025.1 & 0.26 & 0.24 & 0.83 & 0.68 & 0.16 & 0.79 & 0.18 & 0.75 & 0.87 & 1.08 & 1.05 \\
\hline Hentriacontane & 3132.3 & 12.35 & 12.30 & 20.79 & 16.72 & 4.48 & 17.92 & 13.36 & 12.0 & 18.63 & 9.96 & 18.13 \\
\hline Dotriacontane* & & 0.50 & 0.47 & 0.93 & 0.79 & 0.07 & 0.60 & 0.14 & 0.46 & 1.19 & 1.04 & 1.19 \\
\hline Tritriacontane * & & 2.00 & 3.14 & 7.38 & 5.97 & 1.01 & 5.30 & 1.43 & 3.66 & 5.78 & 7.85 & 6.78 \\
\hline Total hydrocarbons: & & 25.57 & 26.61 & 47.24 & 39.34 & 13.33 & 42.13 & 18.09 & 26.50 & 40.21 & 48.27 & 26.55 \\
\hline
\end{tabular}


Components

KIE $\quad \begin{aligned} & \text { Shar Planina } \\ & 20082009\end{aligned}$

Suva Gora Prilepsko**

Bistra

Kozuf Nidze Ilina Mtn. 20082009 20082009 20082009 20082008

2008

\section{FATTY ACIDS}

Dodecanoic acid

Tetradecanoic acid

Hexadecanoic acid

9,12-ocatadecadienoic acid

9,12,15-octadecatrienoic

acid*

Octadecanoic acid *

Total fatty acids:

\section{ALCOHOLS}

2,4-di-tert-butylphenol

Tetradecanol

Phytol (isomer) ${ }^{t^{*}}$

2-Hexadecen-1-ol

Oleyl alcohol

Octadecanol

Total alcohols:

\section{STEROLS}

(-)-Cholesterol*

22,23-dihydrobrassicasterol*

Stigmasterol*

$\gamma$-Sitosterol*

Total sterols:

\section{TRITERPENS}

Squalene*

$\beta$-Amyrine*

$\alpha$-Amyrine*

Triterpene alcohol*t

Total triterpens:

\section{OTHER COMPOUNDS}

Hexahydrofarnesil acetone

TOTAL

$\begin{array}{cccccccccccc}1643.2 & 0.03 & 0.01 & - & - & - & - & - & - & - & & - \\ 1860.6 & 0.04 & 0.02 & - & - & - & - & - & 0.02 & 0.05 & 0.10 & 0.08 \\ 2070.2 & 0.75 & 0.74 & 1.60 & 1.91 & 0.89 & 3.09 & 0.63 & 1.66 & 1.38 & 2.10 & 0.91 \\ 2258.0 & - & - & 0.42 & 1.03 & 0.10 & 1.84 & 0.20 & 0.32 & 1.52 & 1.49 & 0.93 \\ & 0.60 & 0.42 & 0.73 & 1.23 & 0.20 & 3.17 & 0.41 & 1.24 & - & 0.98 & 0.88 \\ & 0.16 & 0.14 & 0.55 & 0.45 & 0.04 & - & - & 0.27 & 0.41 & 0.75 & 0.63 \\ & 1.58 & 1.33 & 3.30 & 4.62 & 1.23 & 8.10 & 1.24 & 3.51 & 3.36 & 5.42 & 3.43\end{array}$

$\begin{array}{lllllllllllllllll}1590.3 & - & - & - & - & - & - & 0 & 0.04 & - & 0.04 & 0.12 & 0.08 & 0.03 & 0.03\end{array}$

$\begin{array}{llllllllll}1765.2 & - & 0.01 & - & - & - & 0.13\end{array}$

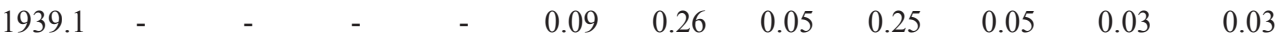

$1981.0 \quad-\quad 0.05 \quad-\quad \begin{array}{llllllllllll} & - & - & 0.03 & 0.12 & - & 0.09 & 0.01 & 0.04 & -\end{array}$

$\begin{array}{llllllllllll}2091.1 & 2.88 & 2.86 & 1.75 & 1.98 & 3.88 & 1.28 & 1.31 & 2.12 & 1.90 & 0.75 & 0.45\end{array}$

$\begin{array}{llllllllllll}2198.8 & 0.23 & 0.21 & 0.08 & - & 0.15 & - & 0.04 & 0.69 & 0.12 & 0.12 & 0.07\end{array}$

$\begin{array}{lllllllllll}3.11 & 3.13 & 2.06 & 1.98 & 3.99 & 1.79 & 1.44 & 3.29 & 2.17 & 0.97 & 0.58\end{array}$

$\begin{array}{ccccccccccc}- & - & - & - & 0.09 & 0.29 & - & 0.29 & 0.26 & 0.28 & - \\ 0.80 & 0.78 & - & - & 0.27 & 0.90 & 0.26 & 0.47 & 0.47 & 0.65 & 0.25 \\ 0.90 & 0.88 & 0.34 & 0.45 & 0.47 & 1.37 & 0.39 & 0.69 & 0.85 & 0.94 & 0.61 \\ - & 1.97 & 1.49 & 1.91 & 1.55 & 3.86 & 0.82 & 2.75 & 1.84 & 3.30 & 2.05 \\ 1.70 & 3.63 & 1.83 & 2.36 & 2.38 & 6.42 & 1.47 & 4.20 & 1.58 & 5.17 & 2.95\end{array}$

$\begin{array}{ccccccccccc}0.17 & 0.15 & 0.48 & 0.54 & 0.40 & 0.66 & 0.07 & 1.01 & 0.52 & 0.66 & 0.37 \\ 0.43 & 0.41 & 0.29 & 0.46 & 0.73 & 0.36 & 0.28 & 0.39 & 0.63 & 2.10 & 0.99 \\ 0.40 & 0.38 & 0.78 & - & 0.40 & 0.41 & 0.19 & 0.28 & 0.35 & 0.25 & 0.30 \\ 0.18 & 0.16 & - & - & \operatorname{tr} & - & 0.01 & - & 0.19 & 0.37 & 0.37 \\ 1.18 & 1.10 & 1.55 & 1.00 & 1.54 & 1.53 & 0.55 & 1.68 & 1.69 & 3.38 & 2.03\end{array}$

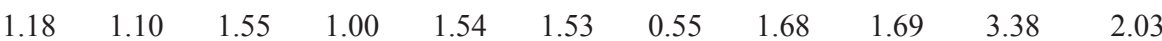

$\begin{array}{rrrrcrrrrrrr}1946.2 & 0.09 & 0.07 & - & - & 0.08 & 0.09 & 0.01 & - & 0.07 & 0.14 & 0.10 \\ & 83.06 & 86.90 & 88.67 & 89.40 & 83.98 & 89.90 & 60.14 & 87.98 & 90.96 & 91.25 & 59.95\end{array}$

* - identification according NIST or Wiley (without AMDIS), ${ }^{\mathrm{t}}$ - tentative identification (AMDIS); ( - )- not found; ( / ) - not aveiable; KIE - estimated retention indices, cultivated plant 
Table 3. Chemical composition of hexane extracts of Sideritis raeseri from different location from Macedonia and Albania

\begin{tabular}{|c|c|c|c|c|c|c|c|c|}
\hline Components & KIE & \multicolumn{2}{|c|}{$\begin{array}{l}\text { Baba Mtn. } \\
\text { Macedonia } \\
20082009\end{array}$} & \multirow[t]{2}{*}{$\begin{array}{c}\text { Galicica } \\
\text { Macedonia } \\
2008\end{array}$} & \multicolumn{2}{|c|}{$\begin{array}{c}\text { Gramoz Mtn } \\
\text { Albania } \\
20082009\end{array}$} & \multirow[t]{2}{*}{$\begin{array}{c}\text { Tepelena } \\
\text { Albania } \\
2008\end{array}$} & \multirow[t]{2}{*}{$\begin{array}{c}\text { Logora Mtn. } \\
\text { Albania } \\
2008\end{array}$} \\
\hline MONO and SESQUITERPENS & & & & & & & & \\
\hline$\alpha$-Pinene & 951.1 & 0.10 & $\operatorname{tr}$ & 0.05 & 0.26 & 0.05 & 0.18 & $\operatorname{tr}$ \\
\hline Camphene & 960.7 & - & - & - & - & - & - & - \\
\hline$\beta$-Pinene & 980.8 & 1.20 & 0,08 & 0.11 & 0.58 & 0.90 & 0.36 & $\operatorname{tr}$ \\
\hline$\beta$-Myrcene & 992.6 & - & - & - & - & - & - & - \\
\hline D-Limonene $+\beta$-Phellandren & 1024.2 & $\operatorname{tr}$ & $\operatorname{tr}$ & $\operatorname{tr}$ & $\operatorname{tr}$ & $\operatorname{tr}$ & $\operatorname{tr}$ & $\operatorname{tr}$ \\
\hline trans-Pinocarveole & 1144.8 & 0.10 & 0,04 & $\operatorname{tr}$ & $\operatorname{tr}$ & $\operatorname{tr}$ & - & $\operatorname{tr}$ \\
\hline cis-Verbenol & 1152.6 & $\operatorname{tr}$ & $\operatorname{tr}$ & $\operatorname{tr}$ & $\operatorname{tr}$ & $\operatorname{tr}$ & - & $\operatorname{tr}$ \\
\hline Myrtenol & 1212.9 & 0.10 & & $\operatorname{tr}$ & 0.02 & 0.04 & - & $\operatorname{tr}$ \\
\hline (-)-Verbenone & 1230.5 & 0.08 & 0,02 & $\operatorname{tr}$ & $\operatorname{tr}$ & $\operatorname{tr}$ & $\operatorname{tr}$ & $\operatorname{tr}$ \\
\hline$\alpha$-Cubebene & 1403.6 & 0.05 & 0,02 & 0.05 & 0.10 & 0.12 & - & - \\
\hline$\alpha$-Copaene & 1437.4 & 0.20 & 0,24 & 0.12 & $\operatorname{tr}$ & 0.10 & 0.05 & 0.04 \\
\hline Muurola-4(14),15-diene (cis) & 1454.2 & - & $\operatorname{tr}$ & 0.08 & $\operatorname{tr}$ & $\operatorname{tr}$ & $\operatorname{tr}$ & - \\
\hline trans-Caryophyllene & 1488.7 & 0.20 & 0,34 & 0.11 & 0.79 & 0.56 & 0.52 & 0.31 \\
\hline Muurola-3,5-diene (trans) & 1524.7 & 0.01 & - & - & - & - & - & - \\
\hline$\alpha$-Hummulene & 1528.9 & 0.10 & 0,12 & 0.06 & 0.04 & 0.06 & $\operatorname{tr}$ & - \\
\hline Germacren D & 1560.7 & 0.02 & 0,06 & 0.06 & 0.06 & 0.02 & 0.02 & 0.04 \\
\hline Bicyclogermacrene & 1578.1 & - & - & 0.08 & - & - & 0.04 & - \\
\hline$\alpha$-Muurolene & 1580.3 & 0.01 & 0,01 & - & - & $\operatorname{tr}$ & - & - \\
\hline$\beta$-Bisabolene & 1588.8 & 0.04 & 0,06 & 0.04 & 0.10 & $\operatorname{tr}$ & - & - \\
\hline$\delta$-Cadinene & 1606.4 & 0.22 & 0,35 & 0.16 & 0.15 & 0.15 & 0.11 & 0.10 \\
\hline Cadina-1,4-diene (trans) & 1617.3 & 0.05 & 0,05 & 0.04 & - & - & - & 0.29 \\
\hline$\alpha$-Calacorene & 1630.2 & 0.02 & 0,02 & 0.03 & - & $\operatorname{tr}$ & - & - \\
\hline (-)-Spatulenol & 1670.2 & $\operatorname{tr}$ & 0,02 & 0.06 & 0.07 & 0.05 & 0.03 & $\operatorname{tr}$ \\
\hline Caryophyllene oxide & 1677.0 & 0.15 & 0,15 & 0.12 & 0.18 & 0.12 & 0.08 & 0.11 \\
\hline Viridiflorol & 1686.1 & 0.09 & 0,24 & 0.08 & 0.06 & 0.10 & - & $\operatorname{tr}$ \\
\hline$\beta$-Gurjunenepoxide & 1690.0 & $\operatorname{tr}$ & 0,01 & - & $\operatorname{tr}$ & $\operatorname{tr}$ & - & $\operatorname{tr}$ \\
\hline $\begin{array}{l}\alpha \text {-Cadinol }+ \\
\tau \text {-Muurolol }\end{array}$ & $\begin{array}{l}1739.1 \\
1739.5\end{array}$ & 0.50 & 1,27 & 0.24 & 0.24 & 0.58 & 0.04 & $\operatorname{tr}$ \\
\hline Valeranone & 1776.5 & 0.05 & 0,05 & 0.11 & - & - & 0.06 & $\operatorname{tr}$ \\
\hline$\alpha$-Bisabolole & 1780.6 & 0.06 & 0,05 & 0.03 & - & - & 0.02 & $\operatorname{tr}$ \\
\hline Total mono and sesquiterpens: & & 3.36 & 3.32 & 1.68 & 2.72 & 3.94 & 1.56 & 1.02 \\
\hline $\begin{array}{l}\text { DITERPENS } \\
(+) \text {-Beyerene }\end{array}$ & 2057.7 & 0.08 & 0,03 & - & 0.05 & 0.03 & 0.05 & 0.04 \\
\hline Pimara-8(14),15-diene & 2072.4 & - & - & - & - & - & - & - \\
\hline Kaur-15-en & 2125.2 & 0.10 & $\operatorname{tr}$ & 0.03 & 0.09 & 0.07 & 0.12 & 0.09 \\
\hline Manoyl oxide & 2145.9 & 0.05 & 0.03 & - & 0.02 & 0.01 & 0.01 & - \\
\hline 3a-Hydroxy manool (M=288) & 2327.5 & 0.20 & 0,21 & 0.10 & 0.33 & 0.37 & 0.47 & 0.23 \\
\hline
\end{tabular}




\begin{tabular}{|c|c|c|c|c|c|c|c|c|}
\hline \multirow[b]{2}{*}{$\begin{array}{l}\text { 7-ethenyl-1- } \\
\text {,2,3,4,4a,4b,5,6,7,9,10,10a-dodeca- } \\
\text { hydro-1,1,4a,7-tetramethyl-2-phenan- } \\
\text { threnol }(\mathrm{M}=306)\end{array}$} & \multirow{2}{*}{$\begin{array}{c}\text { KIE } \\
2335.8\end{array}$} & \multicolumn{2}{|c|}{$\begin{array}{l}\text { Baba Mtn. } \\
\text { Macedonia } \\
20082009\end{array}$} & \multirow{2}{*}{$\begin{array}{c}\text { Galicica } \\
\text { Macedonia } \\
2008 \\
\\
0.07\end{array}$} & \multicolumn{2}{|c|}{$\begin{array}{c}\text { Gramoz Mtn } \\
\text { Albania } \\
2008 \quad 2009\end{array}$} & \multirow{2}{*}{$\begin{array}{c}\text { Tepelena } \\
\text { Albania } \\
2008\end{array}$} & \multirow{2}{*}{$\begin{array}{c}\text { Logora Mtn. } \\
\text { Albania } \\
2008 \\
\\
0.33\end{array}$} \\
\hline & & 0.24 & 0,22 & & 0.21 & 0.24 & & \\
\hline Diterpene (M=306) & 2374.2 & 0.22 & 0,15 & 0.11 & 0.14 & 0.20 & - & 0.27 \\
\hline Diterpene (M=306) & 2387.9 & - & - & - & - & - & - & - \\
\hline Diterpene (M=306) & 2459.0 & - & 0,20 & 0.18 & 0.33 & 0.20 & 0.68 & 0.50 \\
\hline Diterpene ( $\mathrm{M}=286)$ & 2487.7 & 10.36 & 8.99 & 5.40 & 21.07 & 15.87 & 18.22 & 13.16 \\
\hline Diterpene (M=286) & & 17.53 & 11.49 & 3.41 & 18.36 & 18.02 & 25.64 & 23.96 \\
\hline Diterpene ( $\mathrm{M}=346)$ & & 1.20 & 0,20 & 0.08 & 0.65 & 0.45 & 0.25 & 0.21 \\
\hline Diterpen (M=286) & & 1.35 & 0,82 & 0.24 & 1.45 & 1.31 & - & 2.13 \\
\hline Total diterpens: & & 31.31 & 22.35 & 9.62 & 42.73 & 36.65 & 45.81 & 14.92 \\
\hline $\begin{array}{l}\text { ESTERS } \\
\text { Benzyl benzoate }\end{array}$ & 1924.3 & $\operatorname{tr}$ & $\operatorname{tr}$ & 0.02 & - & 0.05 & 0.02 & 0.03 \\
\hline Isopropyl tetradecanoate & 1978.5 & 0.05 & $\operatorname{tr}$ & 0.03 & $\operatorname{tr}$ & 0.35 & 0.03 & 0.03 \\
\hline Di-isobutyl phthalate* & 2029.2 & 0.06 & 0,04 & 0.41 & 0.05 & 0.06 & 0.06 & 0.11 \\
\hline Methyl hexadecanoate & 2103.3 & - & 0,08 & 0.10 & 0.02 & 0.08 & 0.21 & 0.19 \\
\hline Hexadecanoic acid ethylester & 2135.6 & 0.04 & 0,10 & 0.06 & - & 0.12 & 0.20 & - \\
\hline Isopropyl hexadecanoate & 2243.4 & - & - & - & - & - & - & 0.54 \\
\hline Methyl octadecanoate & 1924.3 & 1.35 & 2,54 & 0.07 & 0.62 & 2.86 & 0.06 & - \\
\hline 9,12-Octadienoic acid ethylester & 1978.5 & 0.18 & 0,14 & 0.15 & - & 0.12 & 0.12 & 0.11 \\
\hline Dodecenol acetat * & & & 0,15 & 0.03 & 1.10 & 0.79 & 0.27 & 0.28 \\
\hline Myristyl tetradecanoate * & & 0.12 & 0,17 & 0.18 & 0.04 & 0.26 & 0.09 & 0.23 \\
\hline Total esters: & & 1.81 & 3.24 & 1.05 & 0.84 & 4.69 & 1.06 & 1.52 \\
\hline $\begin{array}{l}\text { HYDROCARBONS } \\
\text { Heptadecane }\end{array}$ & 1790.5 & 0.02 & 0,03 & 0.04 & $\operatorname{tr}$ & 0.04 & - & $\operatorname{tr}$ \\
\hline Octadecane & 1896.4 & 0.02 & 0,01 & 0.02 & $\operatorname{tr}$ & 0.03 & - & $\operatorname{tr}$ \\
\hline Nonadecane & 2002.0 & - & - & - & - & - & - & - \\
\hline Eicosane & 2108.2 & 0.09 & 0,08 & 0.06 & 0.06 & 0.06 & 0.09 & 0.04 \\
\hline Heneicosane & 2213.4 & - & 0,04 & 0.06 & - & 0.04 & 0.07 & 0.04 \\
\hline Docosane & 2318.5 & 0.26 & 0,21 & 0.10 & $\operatorname{tr}$ & 0.02 & 0.15 & 0.15 \\
\hline Tricosane & 2423.2 & 0.41 & 0,21 & 0.17 & 0.40 & 0.34 & 0.43 & 0.36 \\
\hline Tetracosane & 2528.7 & 0.21 & 0,10 & 0.11 & 0.16 & 0.15 & 0.35 & 0.11 \\
\hline Pentacosane & 2631.0 & 0.75 & 0,47 & 0.45 & 0.79 & 0.54 & 0.97 & 0.81 \\
\hline Hexacosane & 2691.5 & 0.08 & 3.52 & 0.05 & 0.13 & 0.11 & 0.17 & 0.15 \\
\hline Heptacosane & 2784.4 & 3.97 & 0.74 & 4.29 & 3.81 & 2.67 & 2.98 & 3.06 \\
\hline Octacosen* & 2810.3 & 1.15 & & 0.63 & 1.16 & 0.78 & 1.66 & 0.97 \\
\hline Octacosane & 2822.2 & 0.53 & 0,66 & 0.74 & 1.08 & 0.83 & 0.92 & 0.53 \\
\hline Nonacosane & 3024.6 & 11.35 & 15.55 & 19.68 & 8.87 & 8.20 & 7.79 & 7.46 \\
\hline Triacontane & 3025.1 & 0.56 & 0,93 & 1.24 & 0.48 & 0.87 & 0.65 & 0.66 \\
\hline Hentriacontane & 3132.3 & 14.50 & 25.40 & 30.31 & 8.09 & 13.25 & 10.39 & 9.14 \\
\hline Dotriacontane* & & 0.72 & 0.97 & 1.35 & - & 0.78 & 0.34 & 0.51 \\
\hline
\end{tabular}




\begin{tabular}{|c|c|c|c|c|c|c|c|c|}
\hline Components & KIE & $\begin{array}{l}\text { Baba } \\
\text { Mace } \\
2008\end{array}$ & $\begin{array}{l}\text { Mtn. } \\
\text { donia } \\
2009\end{array}$ & $\begin{array}{c}\text { Galicica } \\
\text { Macedonia } \\
2008\end{array}$ & $\begin{array}{r}\text { Gram } \\
\text { All } \\
2008\end{array}$ & $\begin{array}{l}\text { z Mtn } \\
\text { ania } \\
2009\end{array}$ & $\begin{array}{c}\text { Tepelena } \\
\text { Albania } \\
2008\end{array}$ & $\begin{array}{c}\text { Logora Mtn. } \\
\text { Albania } \\
2008\end{array}$ \\
\hline Tritriacontane * & & 4.05 & 8.02 & 8.94 & 4.27 & 4.05 & 2.68 & 2.23 \\
\hline Total hydrocarbons: & & 38.67 & 59.94 & 68.12 & 29.33 & 32.76 & 29.64 & 26.24 \\
\hline $\begin{array}{l}\text { FATTY ACIDS } \\
\text { Dodecanoic acid }\end{array}$ & 1643.2 & $\operatorname{tr}$ & $\operatorname{tr}$ & 0.06 & - & - & - & 0.03 \\
\hline Tetradecanoic acid & 1860.6 & 0.06 & 0,02 & 0.10 & 0.04 & 0.05 & - & 0.16 \\
\hline Hexadecanoic acid & 2070.2 & 0.92 & 1,87 & 2.23 & 0.03 & 2.72 & 0.55 & 3.68 \\
\hline 9,12-ocatadecadienoic acid & 2258.0 & 0.40 & 0,54 & 1.80 & 0.55 & 0.68 & 0.06 & 0.83 \\
\hline $9,12,15$-octadecatrienoic acid* & - & - & - & - & - & - & - & 0.30 \\
\hline Octadecanoic acid* & - & - & - & 0.73 & - & - & - & 0.30 \\
\hline Total fatty acids: & & 1.39 & 2.44 & 4.92 & 0.62 & 3.45 & 0.61 & 5.30 \\
\hline $\begin{array}{l}\text { ALCOHOLS } \\
\text { 2-Nonen-1-ol }\end{array}$ & 1072.3 & $\operatorname{tr}$ & 0,02 & $\operatorname{tr}$ & - & - & - & - \\
\hline 2,4-di-tert-butylphenol & 1590.3 & 0.06 & $\operatorname{tr}$ & 0.12 & - & 0.07 & 0.09 & 0.11 \\
\hline Tetradecanol & 1765.2 & 0.12 & 0,17 & 0.24 & 0.05 & 0.17 & 0.07 & 0.25 \\
\hline Phytol (isomer) ${ }^{t}$ & 1939.1 & 0.05 & 0,20 & 0.07 & 0.16 & 0.23 & 0.08 & 0.04 \\
\hline 2-Hexadecen-1-ol & 1981.0 & 0.04 & 0,07 & 0.03 & - & - & - & - \\
\hline Oleyl alcohol * & 2091.1 & 1.82 & 1,04 & 0.28 & 1.51 & 1.23 & 2.07 & 2.80 \\
\hline Octadecanol & 2198.8 & 1.12 & 0,06 & - & 0.07 & 0.06 & 0.10 & \\
\hline Total alcohols & & 3.22 & 1.50 & 0.75 & 1.79 & 1.76 & 2.41 & 3.20 \\
\hline $\begin{array}{l}\text { STEROLS } \\
(-) \text {-Cholesterol* }\end{array}$ & & 0.16 & 0,24 & - & 0.13 & 0.23 & 0.28 & 0.36 \\
\hline 22,23-dihydrobrassicasterol * & & 0.52 & 0,43 & 0.26 & 0.36 & 0.42 & 0.28 & 0.20 \\
\hline Stigmasterol* & & 0.75 & 0,77 & 0.61 & 0.79 & 0.55 & 0.46 & 0.49 \\
\hline$\gamma$-Sitosterol* & & 2.27 & 2,36 & 1.88 & 2.07 & 1.91 & 1.69 & 1.35 \\
\hline Total sterols & & 3.70 & 3.80 & 2.75 & 3.35 & 3.11 & 2.71 & 2.40 \\
\hline $\begin{array}{l}\text { TRITERPENS } \\
\text { Squalene * }\end{array}$ & & 0.36 & 0,48 & 0.52 & 0.45 & 0.52 & 0.31 & 1.41 \\
\hline$\beta$-Amyrine* & & 0.59 & 0,36 & 0.82 & 0.56 & 0.31 & 0.56 & 0.44 \\
\hline$\alpha$-Amyrine* & & 0.39 & 0,28 & 0.23 & 0.57 & 0.41 & 0.53 & 0.42 \\
\hline Triterpene alcohol ${ }^{\mathrm{t}} *$ & & 0.33 & 0,22 & 0.27 & 0.24 & 0.13 & 0.17 & 0.11 \\
\hline Total triterpens & & 1.67 & 2.01 & 1.84 & 1.82 & 1.37 & 1.57 & 2.38 \\
\hline $\begin{array}{l}\text { OTHER COMPOUNDS } \\
\text { Hexahydrofarnesil acetone }\end{array}$ & 1946.6 & - & 0,08 & 0.17 & 0.12 & 0.09 & 0.10 & 0.08 \\
\hline TOTAL & & 85.15 & 98.68 & 90.90 & 83.29 & 80.94 & 85.47 & 83.06 \\
\hline
\end{tabular}

\footnotetext{
* - identification according NIST or Wiley (without AMDIS), ${ }^{\mathrm{t}}$ - tentative identification (AMDIS); ( - )- not found; KIE - estimated retention indices
} 


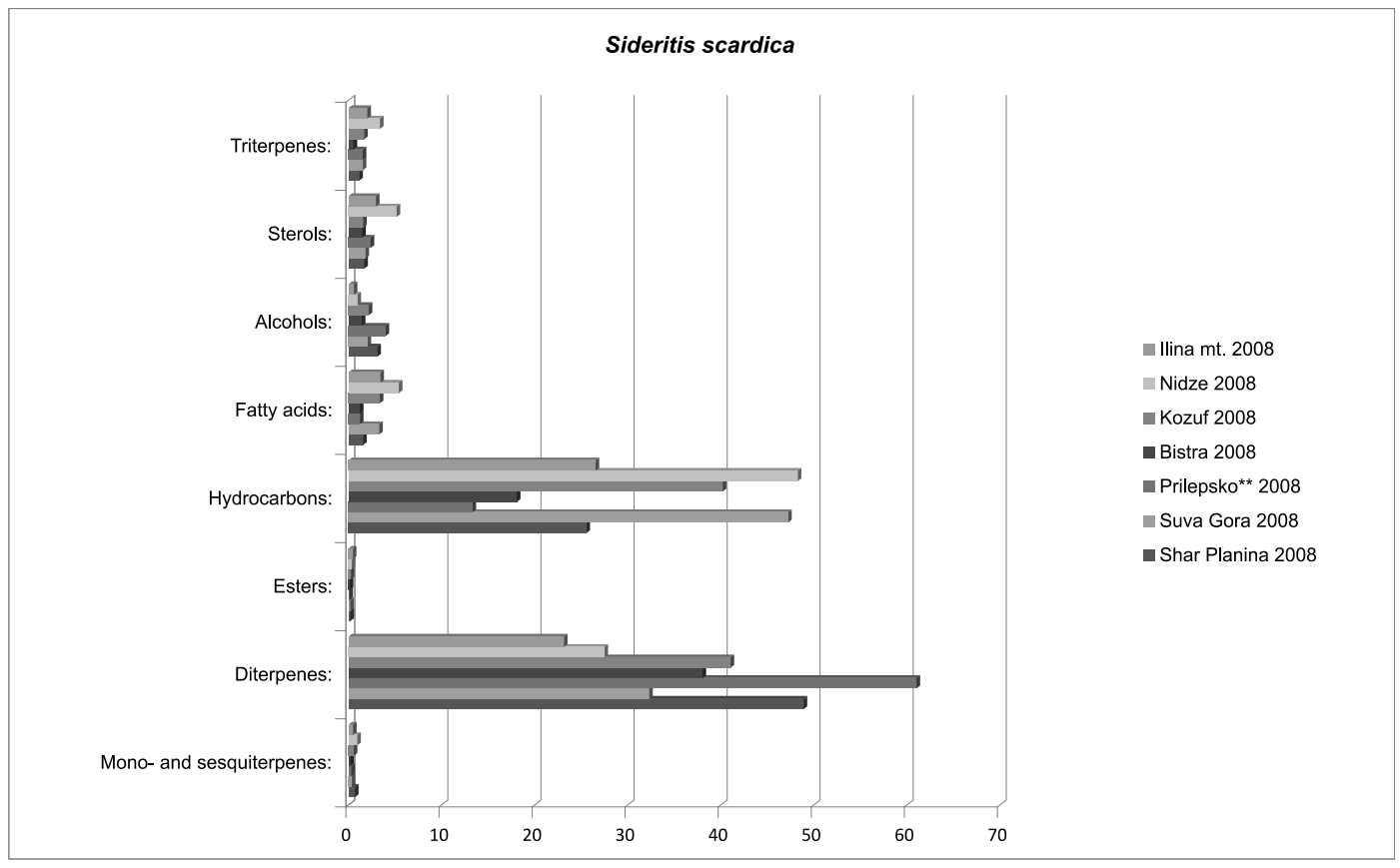

Fig. 1. The content of different types of components in the $n$-hexane extracts of Sideritis scardica

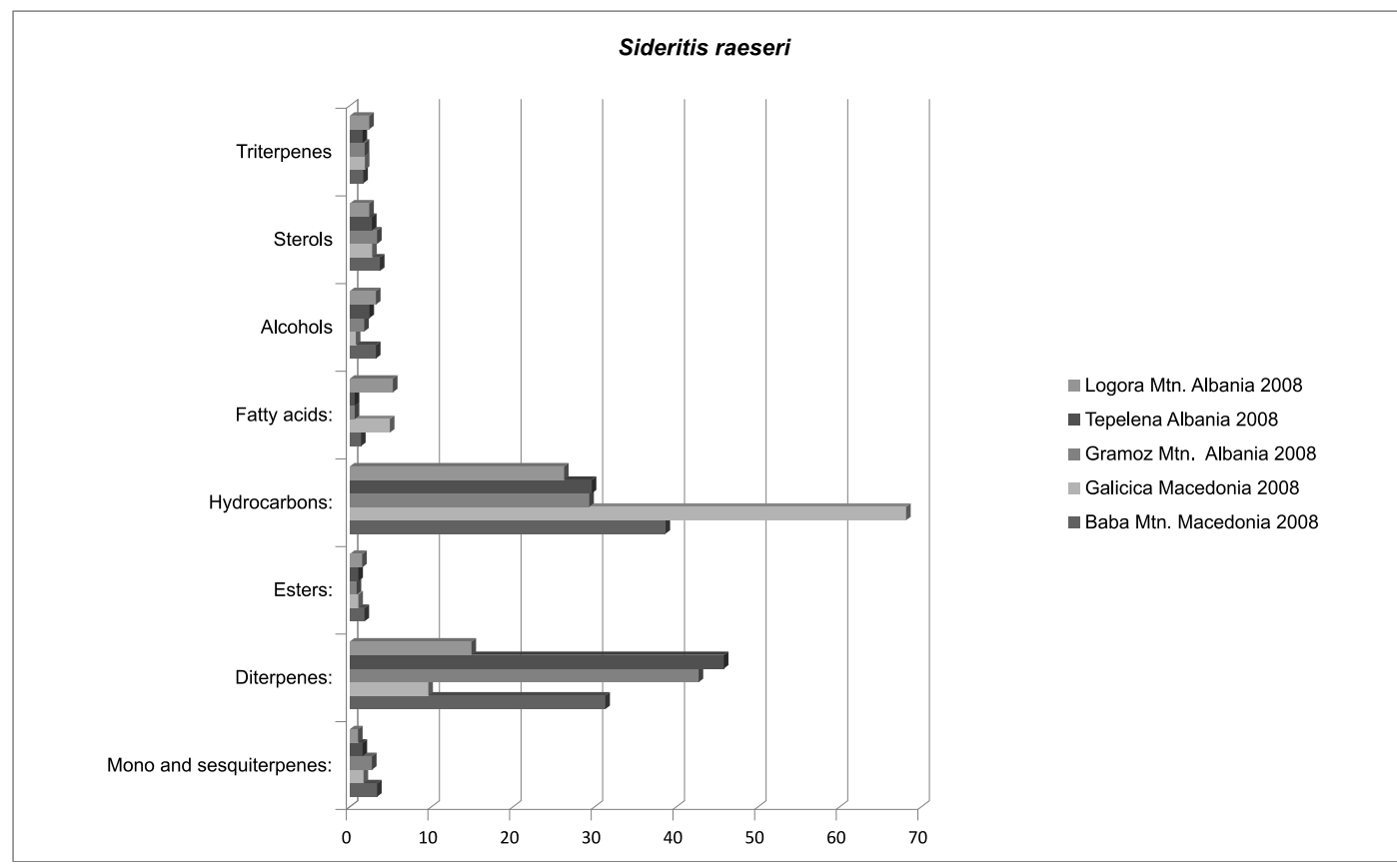

Fig. 2. The content of different types of components in the $n$-hexane extracts of Sideritis raeseri

type of diterpenes (Gomez-Serranillos et al., 1997, 1998; Topku et al., 2001, 2002). Seasonal variation in the content of some diterpenes were registered and the most variable was the most dominant diterpenes, mainly in the $n$-hexane extracts of $S$. scardica (Table 2 and 3).

In $n$-hexane extracts of $S$. scardica and $S$. scardica $S$. syriaca hybride, Kostadinova et al. (2008) found predom- inant presence of diterpene fractions in both species, in amounts from $13.8-54.1 \%$. Ten diterpene components were registered and only three were fully identified (manoyl oxide, 7-ethenyl-1,2,3,4,4a,4b,5,6,7,9,10,10a-dodecahydro1,1,4a,7-tetramethyl-2-phenanthrenol and pimara-8,15dine-3-ol. The other diterpene components had following molar mass: 288, 286, 290, 304 and 346. The most abun- 
dant was $\mathrm{M}=346$ diterpene. Comparing these data with our results, some similarities could be found. We also registered diterpene $\mathrm{M}=346$, but this components was present in a very small amounts in comparison to other components and especially to components with $\mathrm{M}=286$. As there were no other literature data considering diterpene components in $S$. scardica and $S$. raeseri, we made some comparations with other Sideritis species. Thus, Topcu et al. (2001) have defined ten diterpenees in Sideritis argyrea: candol, 7-epicandicanol, ent-7 $\alpha$-acetoxy-18-hydroxykaur-16-ene, foliol, linearol, sidol, 7-epocandicandiol 18-monoacetate, siderol and 11,12-sideridiol, all of them with kaurane skeleton and only one labdane diterpene named ent- $6 \alpha, 8 \alpha-$ dihydroxylabda-13(16)14-diene. Later, the same research group published that, besides above mentioned components, Sideritis sipilea and S. dichotoma contain also different kaurane isomers, but also some beyerne components (Topku et al., 2002). In Iberian's Sideritis species, GomezSerranilos et al. (2004) determined the content of ten diterpenes: serradiol, linearol, conchtriol, foliol, isofoliol, andalusol, lagascatriol, tobarrol, sidol and siderol, seven of them kauranes.

In general, diterpenes represented very interesting group of components because of their chemistry and their distribution in plants, the possibilities for their use as markers in order to find or approve some taxonomy solutions, but much more because of their possible biological and pharmacological activity and further use in healing illnesses or other use. For these reasons, diterpenees of Siderits scardica and $S$. raeseri should be further more investigated, for full identification and determination and assessment of possible biological activity.

\section{Conclussion}

The ultrasonic-assisted extraction process with $n$ hexane on dried over-ground parts of two species of Sideritis collected on different locations in western part of Macedonia and south-eastern part of Albania, yielded 0.73$3.33 \%$ and $9.11-10.44 \%$ of extracts for S. scardica Grieseb. and $S$. raeseri Boiss. \& Heldr., respectively. Over one hundred constituents of the extracts were identified using gas-chromatography (FID) and gas-chromatography/mass spectrometry methods. The components belonged to several classes of components such as diterpenes and hydrocarbons, dominantly presented in each of the extracts, followed by fatty acids, aliphatic and aromatic alcohols, sterols, triterpene alcohols and different monoterpenes and sesquiterpenes, found in much smaller amounts or traces. The most abundant constituents of the extracts of both species of Sideritis were two diterpenee components, both with $\mathrm{M}=286$, which were not fully identified. Large percentages of nonacosane (1.71-12.22\% and 7.46-19.68\% for S. scardica and S. raeseri, respectively) and hentriacontane (4.48-20.79\% and 8.09-30.31\% for S. scardica and S. raeseri, respectively) were also found in the extracts of both species. Season- al variation in the content of some diterpenes and hydrocarbones was found for the both species of Sideritis.

\section{References}

Aboutabl, E.A., Nassar, M.I., Elsakhawy, F.M., Maklad, Y.A., Osman, A.F., El-Khrisy, E.A.M., 2002. Phytochemical and pharmacological studies on Sideritis taurica Stephan ex Wild, J. Ethnopharmac. 82, 177-184.

Alcaraz, M.J., Jimenez, M.J., Valverde, S., Sanz, J., Rabanal, R.M., Villar, A., 1989. Anti-inflammatory compounds from Sideritis javalambrensis n-hexane extract, J. Nat. Prod. 52 (5), 1088-1091

Alipieva, K., Petreska, J., Gil-izquierdo, A., Stefova, M., Evstatieva, L., Bankova, V., 2010. Influence of the extraction method on the yeald of flavonoids and phenoluics from Sideritis spp. (Pirin Mountain tea), Nat. Prod. Commun. 5, 51- 54.

Alipieva, K.I., Kostadinova, E.P., Evstatieva, L.N., Stefova, M., Bankova, S.V., 2009. An iridoid and a flavonloid from Sideritis lanata L., Fitoterapia 80 (1), 51- 53.

Armata, M., Gabrieli, C., Termentzi, A., Zervou, M., Kokkalou, E., 2008. Constituents of Sideritis syriaca ssp. syriaca (Lamiaceae) and their antioxidant activity, Food Chemistry $111,179-186$

Erkan, N., Cetin, H., Ayranci, E., 2011. Antioxidant activities of Sideritis congesta Davis et Huber-Morath and Sideritis arguta Bois et Heldr: Identification of free flavonoids and cinnamic derivatives, Food Research International 44, 297-303.

Gabrieli, C.N., Kefalas, P.G., Kokkalou, E.L., 2005. Antioxidant activity of flavonoids from Sideritis raeseri, J. Ethnopharmac. 96, 423-428.

Gomez-Serranillos, M.P., Carretero, E., Slowing, K., Palomino, O.M., Villarrubia, A.I., Villar, A., 1998. HPLC quiantitative analysis of diterpeneoids in Sideritis (Labiatae) species, Phytoth. Res. 12, 101-103.

Gomez-Serranillos. M.P., El-Nager. T., Villar. A.M., Carretero. M.E., 2004. Analysis of retention behavior in highperformance liquid chromatography of terpenic plant constituents (Sideritis spp.) with pharmacological interest, J. Chromatogr. B, 812, 379-383.

Gomez-Serranillos M.P., Palomino O.M., Villarrubia A.I., Cases M.A., Carretero E., Villar A., 1997. Analysis of diterpeneoids from Sideritis species by reversed-phase high-performance liquid chromatography, J. Chromatogr. A, 778, 421-425.

Janeska, B., Stefova, M. Alipieva, K., 2007. Assay of flavonoid aglycones from the species of genus Sideritis (Lamiaceae) from Macedonia with HPLC-UV DAD, Acta Pharm. 57, 371-377.

Koleva, I.I., Linssen, J.P.H., van Beek, T.A., Evstatieva, L.N., Kortenska, V., Hadjieva, N., 2003. Antioxidant activity screening of extracts from Sideritis species (Labiatae) grown in Bulgaria, J. Sci. Food. Agric., 83, 809-819.

Kostadinova, E., Alipieva, K., Stefova, M., Antonova, D., Evstatieva, L., Stefkov, G., Tsvetkova, I., Naydenski, H., Bankova, V., 2008. Influence of cultivation on the chemical composition and antimicrobial activity of Sideritis spp., Pharmacog. Magazine 4, 102-106.

Kostadinova, E., Nikolova, D., Alipieva, K., Stefova, M., Stefkov, G., Evstatieva, L., Matevski, V., Bankova, V., 2007. Chemical constituents of essential oils of Sideritis scardica Griseb. and Sideritis raeseri Boiss. \& Heldr. From Bulgaria 
and Macedonia, Nat. Prod. Res., 21 (9), 819-823.

Lysyansky, V.M., Grebenyuk, S.M., 1987. Extraction in food industry, Agropromizdat, Moskow, pp. 188.

Menghini, L., Massarelli, P., Bruni, G., Menghini, A., 2005. Preliminary evaluation on anti-inflamatory and analgesic effects of Sideritis syriaca L. herba extracts, J. Med. Food 8 (2), 227-231.

Ozkan, G., Sagdic, O, Ozcan, M., Ozcelik, H., Unver, A., 2005. Antioxidant and antibacterial activities of Turkish endemic Sideritis extracts, Grasas y Aceites 56, 16-20.

Palommino, O.M., Gomez-Serranillos, P., Carretero, E., Villar, A., 1996. High-performance liquid chromatography of flavonoids from Sideritis species, J. Chromatog. A, 731, 103-108.

Petreska, J., Stefkov, G., Kulevanova, S., Alipieva, K., Bankova, V., Stefova, M., 2011. Phenolic compounds of Mountain tea from Balkans: $\mathrm{LC} / \mathrm{DAD} / \mathrm{ESI} / \mathrm{MS}^{\mathrm{n}}$ profile and contents, Nat. Prod. Comm. 6, 1-10.

Petreska, J., Stefova, M., Ferreres, F., Moreno, D.A., TomasBarberan, F.A., Stefkov, G., Kulevanova, S., Gil-Izquierdo, 2011. Potential bioactive phenolics of Macedonian Sideritis species used for medicinal "Mountain tea", Food Chemistry 125, 13-20.

Pljevljakusic, D., Savikin, K., Jankovic, T., Zdunic, G., Ristic, M., Godjevac, D., Konic-Ristic, A., 2011. Chemical properties of the cultivated Sideritis raeseri Boiss. \& Heldr. subsp. raeseri, Food Chemistry 124, 226-233.

Rodriguez-Garcia, I., Munoz-Dorado, M., Gomez-Mersado, F., Garcia-Maroto, F., Guill-Guerrero, Jl., 2004. Essential oil composition of Sideritis pusilla (Lange) Pau ssp., J. Essent. Oil. Res. 16, 535-538.

Semagina, N.V., Sulman, M.G., Sulman, E.M., Ankudinova T.V., 2000. Study of ultrasound-stimulated extraction of biologically active substances from row plant materials, Pharma. Chem. J. 34 (2), 69-72.

Sulman, M.G., Pirog, E.M., Ankudinova, T.V., Sulman, E.M., Semagina, N.V., 1997. The extraction process from the vegetable row materiasl in the ultrasonic field, $1^{\text {st }}$ European Congress on Chemical Engineering: Florence, Italy, vol. 4, p. 3017-3018.

Topku, G., Goren, A.C., Kilic, T., Kemal, Y., Tumen, G., 2001. Diterpenees from Sideritis argyrea, Fitoterapia 72, 1-4.

Topku G., Goren, A.C., Kilic, T., Kemal, Y., Tumen, G., 2002. Diterpenees from Sideritis sipylea and S. dichotoma, Turk. J. Chem. 26, 189-194.

Tsaknis, J., Lalas, S., 2005. Extraction and identification of natural antioxidants from Sideritis euboea (mountain tea), J. Agric. Food. Chem. 53 (16), 6375- 6381.

\title{
Хемиски состав на $\boldsymbol{n}$-хексански екстракти добиени со
} ултразвучна ектракција од Sideritis scardica Griseb. и Sideritis raeseri Boiss. \& Heldr. (Lamiaceae) од Македонија и Албанија

\author{
Бујар К'азими, Марија Карапанџова*, Ѓше Стефков, Светлана Кулеванова
}

\author{
Институт за Фармакогнозија, Фармацевтски Факултет, Универзитет Св. Кирил и Методиј, Скопје, Република \\ Македонија
}

Клучни зборови: Sideritis scardica, Sideritis raeseri, n- хексански екстракти, ултразвучна екстракција, состав, GC-MS анализа.

Хемиски состав на $n$-хексански екстракти добиени од суви надземни делови од два вида Sideritis, S. scardica Griseb. и S. raeseri Boiss. \& Heldr. (Lamiaceae) е испитуван со помош на гасна хроматографија (GC/FID) и гасна хроматографија/масена спектрометрија $(\mathrm{GC} / \mathrm{MS})$. Собирањето на растенијата е извршено на различни локалитети во западниот дел на Македонија и во јужниот дел на Албанија, вкупно дванаесет различни примероци на растителен материјал. Ултразвучната екстракција е користена за подготовка на $n$-хексански екстракти, а добиениот принос се движи од 0,73-3,33\% и 9,11-10,44\% на екстракти на S. scardica, односно S. raeseri. Во екстрактите се идентификувани повеќе од сто компоненти, кои што припаѓаат на неколку класи соединенија како што се дитерпени и јаглеводороди, главно присутни во секој екстракт, проследени со масни киселини, алифатични и ароматични алкохоли, стероли, тритерпенски алкохоли и разни монотерпени и сесквитерпени, што се најдени во многу помали количества или само во траги. Најзастапени компоненти на екстрактите од двата видови Sideritis се две дитерпенски компоненти, двете со М $=286$, кои што не се целосно идентификувани. Голем процент од нонакозан (1,71-12,22\% и 7,46-19,68\% за $S$. scardica, односно $S$. raeseri) и хентриаконтан (4,48 - 20,79\% и 8,09-30,31\% за S.scardica, односно S.raeseri) се најдени во екстрактите од двата вида. 